Document downloaded from:

http://hdl.handle.net/10251/103131

This paper must be cited as:

Benítez-González, J.; Mora Almerich, J.; Bolea Boluda, M. (2017). SCM Adaptation to Improve Scanning Rate in RF Interferometry Applications. IEEE Photonics Technology Letters. 29(12):999-1002. doi:10.1109/LPT.2017.2700884

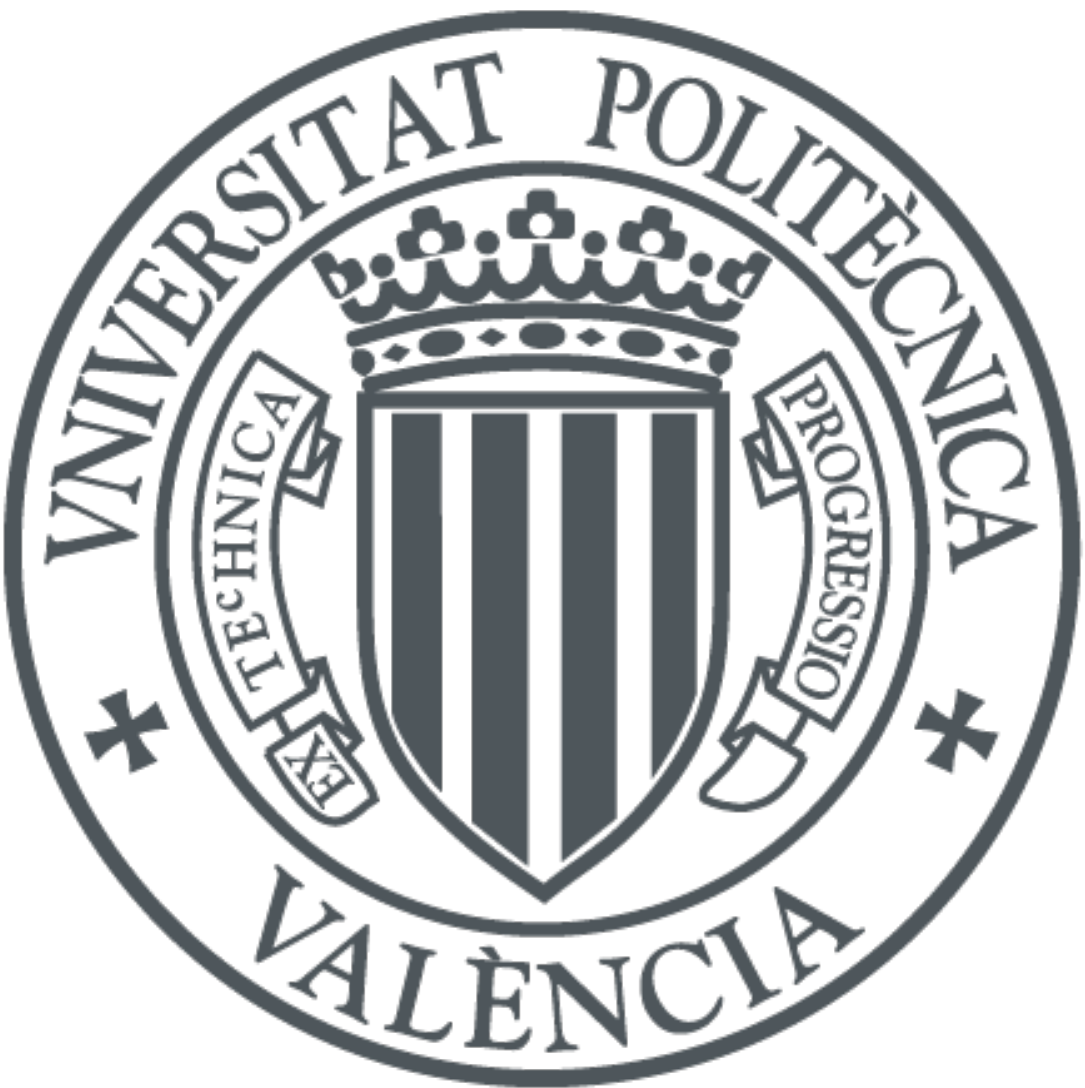

The final publication is available at

https://doi.org/10.1109/LPT.2017.2700884

Copyright Institute of Electrical and Electronics Engineers

Additional Information 


\title{
SCM adaptation to improve scanning rate in RF interferometry applications
}

\author{
J. Benítez, J. Mora, M. Bolea
}

\begin{abstract}
In this paper, we present a novel structure for performing subcarrier multiplexing (SCM) to improve the scanning rate in low coherence interferometry (LCI) systems combined with the microwave photonics (MWP) technology. In this MWP-LCI proposal, the optical path differences (OPDs) produced by different samples between the arms of an interferometer are closely related to the central frequency of different $R F$ resonances generated in the $R F$ domain. By the proposed adaptation of the SCM technique, 'M' subcarriers are multiplexed in the modulation stage and each subcarrier is set to sweep simultaneously a concrete part of the spectrum. The complete electrical transfer function of the structure is obtained combining each individual sweep. Therefore, a considerable reduction of the sweep time is provided to collect the complete electrical transfer function. Therefore, the scanning rate is reduced according to the number of subcarriers $(M)$ employed in the multiplexing stage. An OPD range of $8 \mathrm{~mm}$ is achieved with a constant resolution of $120 \mu \mathrm{m}$ in the whole range. Finally, a maximum sensitivity of $60 \mathrm{~dB}$ is also reached for that operation range.
\end{abstract}

Index Terms - Low coherence interferometry, microwave photonics, subcarrier multiplexing, optical path difference, scanning rate.

\section{INTRODUCTION}

L OW coherence interferometry (LCI) is a well-known measurement technique able to provide an excellent axial positioning precision. Its development through the last decades has been demonstrated in a high number of publications [1-5], mainly focused in the medical field, where the optical coherence tomography (OCT) constitutes the most important research area [1,2]. Nevertheless, LCI can be applied to a wide number of fields such as art conservation [3], components characterization [4] or sensing [5]. Its basic principle consists in the characterization of the interference pattern produced by the combination of the scattered light illuminating a sample and the reflection in a reference mirror. By employing an incoherent source, it is possible to determine the path that light has travelled in the case of each beam and precisely resolve the position of the different events produced

Manuscript received March 24th, 2017. The research leading to these results has received funding from the National Project TEC2014-60378-C2-1$\mathrm{R}$ funded by the Ministerio de Ciencia y Tecnología and the regional project PROMETEO FASE II/2013/012 funded by the Generalitat Valenciana.

J Benítez, J. Mora and M. Bolea are with the ITEAM Research Institute, Universitat Politècnica de València, Valencia, Spain (e-mail: imalmer@iteam.upv.es). in the interferogram.

Current LCI schemes are based on the optical processing by means of interferometric structures. Consequently, the interference pattern generated in the optical domain is affected by environmental variations, what brings instability to the system performance. In order to address this issue, the cost and reliability of the schemes are increased by means of a huge design complexity. In this way, microwave photonics (MWP) constitutes an excellent approach to solve this issue. MWP is a technology which main objective is to improve the capabilities of radiofrequency (RF) engineering by its combinations with the optical technology [6]. Although its evolution has been dependent on the development of the communication field, MWP has been related to more diverse areas which include terahertz-wave generation [7] or noninvasive high resolution sensing [8].

Combination of MWP and LCI (MWP-LCI) has become an attractive solution as can be seen in some recent publications [9-13]. Different proposals can be found where the slicing of an incoherent source and its analysis through a dispersive element has been demonstrated to retrieve the visibility of low-coherence interferograms by measuring the electrical transfer function in the RF domain [9-11]. Nevertheless, the scanning rate of these proposals is restricted to the sweep speed of commercial equipments [13]. In order to achieve high scanning rates, time domain techniques including high speed oscilloscopes have been proposed [12]. Comparing optical and RF domain, the inherent stability of RF domain techniques is a relevant advantage to improve LCI key parameters such as resolution and sensitivity [13].

In this context, a MWP-LCI scheme is proposed to operate in the RF domain by means of subcarrier multiplexing (SCM) technique in order to reduce the total scanning rate. Therefore, the time reduction is proportional to the number of subcarriers employed in the multiplexing. Our proposal reaches OPD ranges of $8 \mathrm{~mm}$, maintaining a constant resolution of $120 \mu \mathrm{m}$. The maximum sensitivity value obtained in this case is $60 \mathrm{~dB}$.

\section{SYSTEM PERFORMANCE WITH SCM ADAPTATION}

The layout of the MWP-LCI employing SCM technique is shown in Fig. 1. Firstly, an incoherent optical source is double-sideband modulated by means of an intensity electrooptic modulator (MOD). In this case, ' $\mathrm{M}$ ' radio frequency subcarriers $\left(\Omega_{1}, \Omega_{2}, \ldots, \Omega_{\mathrm{M}}\right)$ are multiplexed (MUX) and introduced in the modulator. According to inset (a) of Fig. 1, a uniform optical profile with $8.8 \mathrm{~nm}$ bandwidth is generated by 


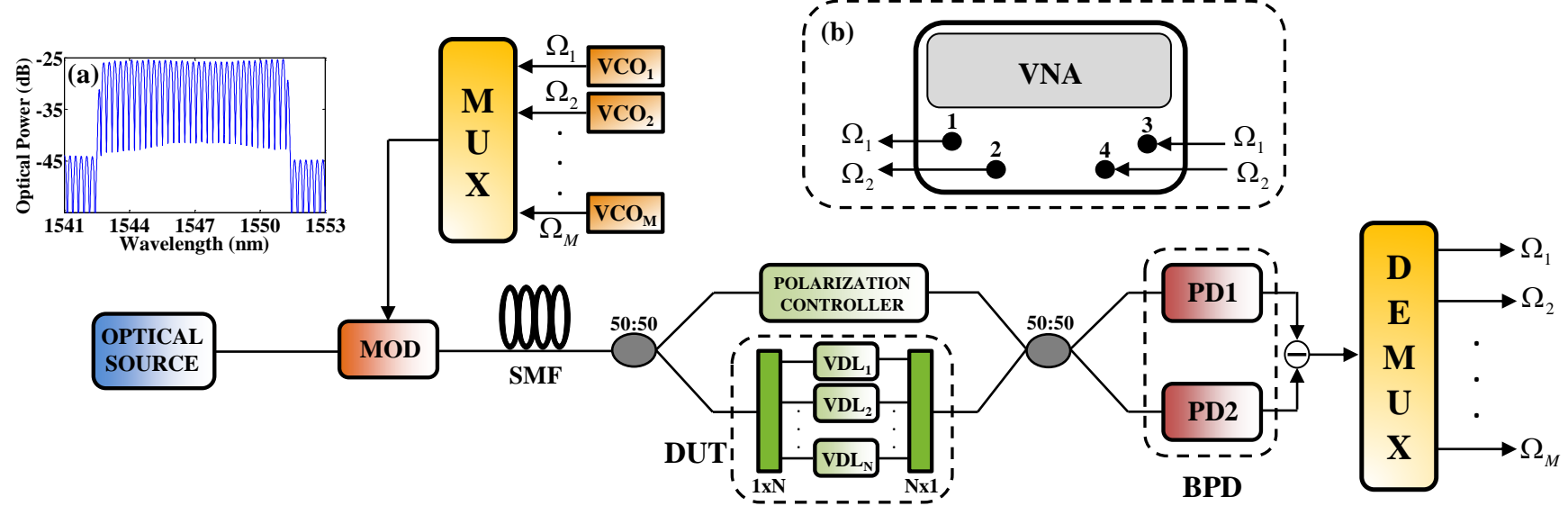

Fig. 1. Layout of the proposed MWP-LCI structure employing SCM. Inset (a) represents the uniform optical profile employed in the experimental setup. Inset (b) represents how the VNA (Agilent E8364A) is set for the experimental measurements.

employing a broadband source (BBS) and an optical channel controller (OCS) [10]. The BBS has a total bandwidth of 80 $\mathrm{nm}$. The OCS is used as an equalizer to flatten the power spectral density of the BBS. It is capable of attenuating in the $1527.60-1565.50 \mathrm{~nm}$ range by means of 48 channels spaced at $100 \mathrm{GHz}$.

In order to show the system performance, two RF subcarriers $\left(\Omega_{1}, \Omega_{2}\right)$ are considered for introducing SCM technique in our MWP-LCI scheme. Experimentally, we use a vector network analyzer (VNA) provided with four RF ports as shown in inset (b) of Fig. 1. On one hand, two RF ports are configured to generate both RF subcarriers which are multiplexed previously to the MOD. On the other hand, two RF ports are used to measure the corresponding electrical transfer function after demultiplexing both RF subcarriers. Note that the number of RF subcarriers can be considerably improved by employing voltage controlled oscillators (VCO) as replacement for the multiport VNA.

After the modulation process, the optical signal is launched into a dispersive element characterized by an accumulated dispersion of $\ddot{\varphi}_{2}$. Concretely, a single mode fiber (SMF) is considered, with an accumulated dispersion of $\ddot{\varphi}_{2}=-440 \mathrm{ps}^{2}$. Then, a Mach-Zehnder interferometer (MZI) is placed in the scheme by means of two 50:50 fiber couplers. In the lower arm, the device under test (DUT) is placed. In the upper arm, a polarization controller device is employed to ensure the maximum visibility of the interference pattern. This interferometer generates the slicing of the modulated optical signal which is related to the optical path difference (OPD) of the optical sample used as DUT. Finally, the output fiber coupler of the MZI is connected to the balanced photodetector (BPD). The RF signal detected is produced as the difference between the contributions in PD1 and PD2. This RF signal obtained is finally demultiplexed (DEMUX) in order to analyze the electrical transfer function of the system attending to each one of the subcarriers $\left(\Omega_{M}\right)$.

The operation principle of MWP-LCI systems is based on the measurement of OPDs by means of the RF system response. According to [14], the electrical transfer function of the system shows a RF resonance centered at the RF frequency $\Omega_{0}$ :

$$
H_{R F}(\Omega) \approx \int S(\omega) \cdot e^{j \ddot{\varphi}_{2} \omega\left(\Omega-\Omega_{o}\right)}
$$

Note that the electrical transfer function $H_{R F}(\Omega)$ is directly related to the power spectral density $S(\omega)$ of the optical source. Therefore, the BBS has to be reconfigured to optimize the RF response. Moreover, the measurement of the central frequency from the RF resonance is proportional to the OPD of the sample (DUT) as follows:

$$
\Omega_{o}=\frac{2 \cdot O P D}{c \cdot\left|\ddot{\varphi}_{2}\right|}
$$

This MWP-LCI operates under small signal modulation. Therefore, the modulation stage reduces drastically the crosstalk due to interference between RF subcarriers. Generalizing the theoretical development described in [14], for this structure and considering the possibility of a n-layered sample, different RF resonances are generated as a result of the interferences produced in the MZI structure.

In order to consider an $n$-layered sample, ' $n$ ' variable delay lines (VDL) are introduced by concatenating two $1 \mathrm{xN}$ star couplers as shown Fig.1. Each VDL is set to generate different OPDs to emulate a multi-layered real sample. For the DUT, a two-layered sample scenario is considered in the lower arm of the interferometric structure. Consequently, two VDLs are employed in the structure. The experimental results obtained are shown in Fig. 2 along with theoretical simulations.

$\mathrm{VDL}_{1}$ is set to produce an $\mathrm{OPD}_{1}$ of $2.1 \mathrm{~mm}$, while $\mathrm{VDL}_{2}$ is configured to an $\mathrm{OPD}_{2}$ of $6 \mathrm{~mm}$. As consequence of the OPDs set in the VDLs, two RF resonances can be observed in Fig. 2, around $5 \mathrm{GHz}\left(\mathrm{OPD}_{1}=2.1 \mathrm{~mm}\right)$ and around $15 \mathrm{GHz}\left(\mathrm{OPD}_{2}=\right.$ $6 \mathrm{~mm}$ ). The amplitudes of the RF resonances are normalized to the peak value of the resonance located at baseband, i.e., low RF frequencies. Due to the performance of the multiplexing technique, two electrical transfer functions are simultaneously obtained as depicted in Fig. 2. On one hand, one port of the VNA is configured to generate an RF sweep between $10 \mathrm{MHz}-10 \mathrm{GHz}$. Fig. 2(a) shows the RF response corresponding to the subcarrier $1\left(\Omega_{1}\right)$. On the other hand, the second subcarrier $\left(\Omega_{2}\right)$ is multiplexed in the modulation stage with a RF sweep form $10 \mathrm{GHz}$ to $20 \mathrm{GHz}$ as shown in Fig. 


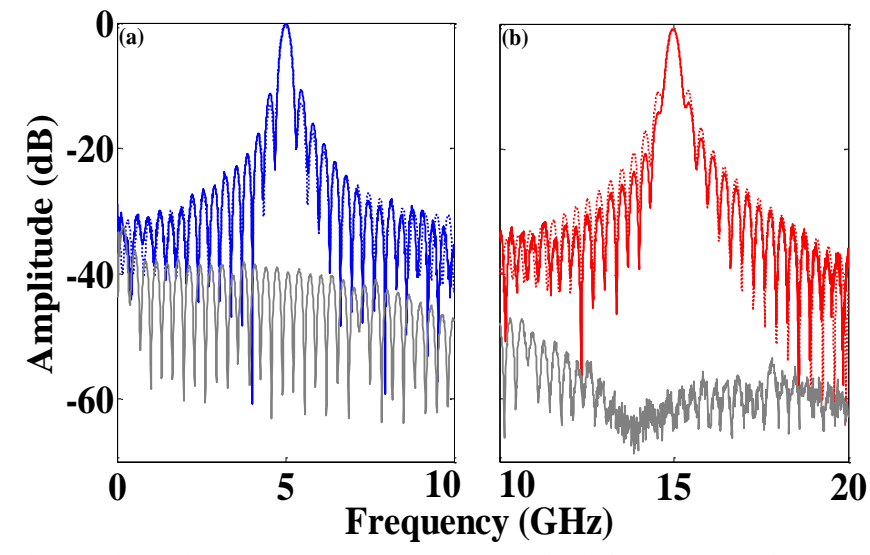

Fig. 2. Theoretical results simultaneously obtained for (a) subcarrier 1 and (b) subcarrier 2, along with experimental results (dashed line). Measurement of the electrical transfer function when the sample is not present in the scheme (noise contribution) has been added in grey line.

2(b). Comparing with previous MWP-LCI approaches [9-11], our proposal highly reduces the baseband contribution located at low RF frequencies due to the BPD as shown Fig. 2(a).

In addition, the electrical transfer function when the sample is not present in the system has been measured. Experimental results and theoretical simulations can be seen in Fig. 2 (grey line), also normalized to the peak value of the RF resonance at baseband. This response is considered as noise for the structure. Therefore, the sensitivity is limited by this contribution. In LCI applications, sensitivity is generally defined as the lower reflectivity coming from the sample that can be detected by the system, i.e., when the value of the signal-to-noise ratio (SNR) is equal to $\mathrm{SNR}=1$ [2]. Note that for this specific scenario with two subcarriers, the total sweep time is reduced to half the value. As abovementioned, the sweep time can be easily extended to a factor ' $M$ ' when considering ' $\mathrm{M}$ ' VCOs in the mux/demux stages.

\section{EXPERIMENTAL RESULTS}

In this section, the system is analyzed through different experimental considerations. We show the inherent stability and the lack of crosstalk of the MWP-LCI structure employing SCM technique in a two-layered sample scenario. Moreover, the system capabilities in terms of LCI parameters (penetration depth, resolution and sensitivity) when considering a one-layer sample scenario are also addressed.

In Fig. 3, two VDLs are considered to measure the corresponding electrical transfer functions. The OPD at $\mathrm{VDL}_{1}$ is set to different values in the range of $0-4 \mathrm{~mm}$ in steps of 0.8 $\mathrm{mm}$ while the OPD at $\mathrm{VDL}_{2}$ has been kept constant to $6 \mathrm{~mm}$. Simultaneously, the electrical transfer function for subcarrier 1 and subcarrier 2 have been collected using the VNA. The electrical response is normalized respect to the electrical response of the MOD and BPD and to the peak value of the resonance located at baseband. Theoretical simulations of the electrical transfer functions for each case are added to Fig. 3. Different RF resonances can be observed in Fig. 3(a) as consequence of the OPDs set in $\mathrm{VDL}_{1}$. In Fig. 3(b), the amplitude of the RF resonance centered at $15 \mathrm{GHz}$ remains constant for any variation in $\mathrm{VDL}_{1}$. This is an indication that

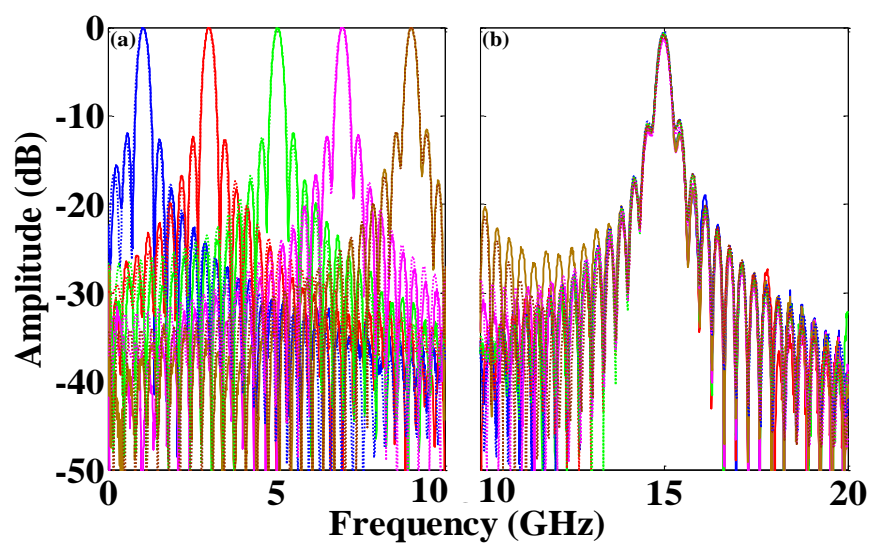

Fig. 3. Electrical transfer functions obtained in the VNA for (a) subcarrier 1 and (b) subcarrier 2 when different OPDs are set in the $\mathrm{VDL}_{1}$. Theoretical simulations have been added in dashed line.

the stability is inherently achieved as known in MWP based systems. Additionally, it also implies that the crosstalk is not relevant as expected since our MWP-LCI system operates under small signal regime. This condition is typically considered in MWP systems such as CATV or QKD since the harmonic distortion and intermodulation degrades the system performance for a high number of subcarriers [15]. For real implementations, we can compare our MWP-LCI system with previous MWP systems. A number up to 50 subcarriers is plausible for the modulation stage considering a crosstalk level which does not reduce the system sensitivity [15]. Therefore, our modulation stage reduces the crosstalk due to interference between RF subcarriers with a potential increase of the scanning rate at least one order of magnitude.

In the following, typical LCI capabilities of the proposed structure are shown for a one-layered sample scenario. First, the OPD is continuously set to different values and the central frequency of the resonances is measured. Theoretical simulation coming from (2) and experimental results can be observed in Fig. 4(a). An excellent linear behaviour in the central frequency displacement of each resonance is observed, proportional to the OPD set in the $\mathrm{VDL}_{1}$. In this case, a slope of $2.47 \mathrm{GHz} / \mathrm{mm}$ and a maximum penetration depth of $8 \mathrm{~mm}$ are achieved. Note that higher penetration depths can be obtained by increasing the accumulated dispersion for the same RF frequency range. An excellent agreement has been obtained between theoretical and experimental results.

Furthermore, the resolution of the system is analyzed in Fig. 4(b). For this, the $3 \mathrm{~dB}$ bandwidth of the RF resonances when setting different OPDs at the VDL are measured in order to determine the resolution through the following equation [10]: $\delta z=-c_{0} \cdot \pi \cdot \ddot{\varphi}_{2} \cdot \delta f_{\text {elec }}$, where $c_{0}$ is the speed of light in vacuum. The theoretical value for the resolution and the experimental results are plotted in Fig. 4(b). An excellent agreement between theoretical and experimental results is achieved, with a maximum resolution value of $120 \mu \mathrm{m}$. For high OPDs, a slight difference is found between the experimental and theoretical results of Fig. 4(b). A quasiinvariant resolution behaviour implies that third-order dispersion can be considered as negligible in our operation range [9]. Therefore, we demonstrate a great control of the 


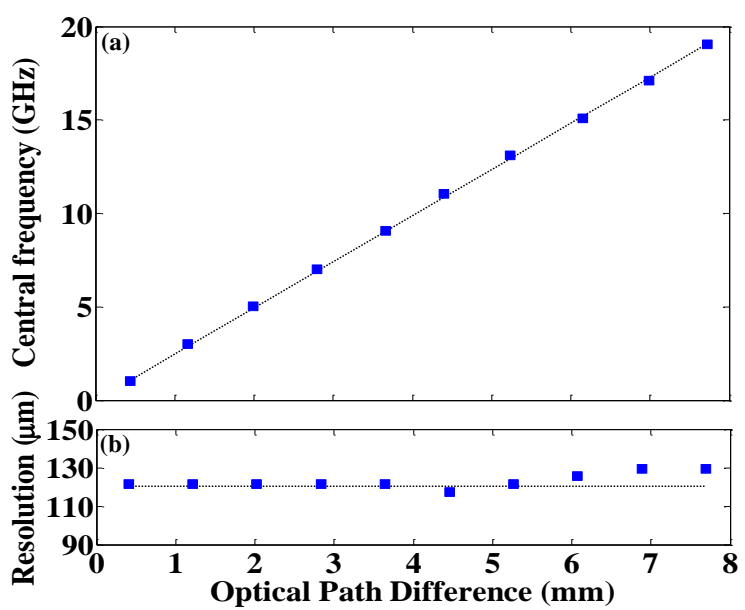

Fig. 4. Experimental (dots) and theoretical (dashed line) results for (a) the central frequency of the RF resonance and (b) the corresponding resolution values when different OPDs are configured in the VDL.

parameters in the whole experiment in comparison to previous MWP-LCI results.

Finally, sensitivity of the system is evaluated in Fig. 5. For this, different OPDs have been set in the VDL of the interferometer and the amplitude of the RF resonances is measured. The noise contribution for obtaining the system sensitivity is shown in Fig. 2. In Fig. 5, we can differentiate two clear ranges in the sensitivity. For the first range from 0 to $5.5 \mathrm{~mm}$, the noise affecting sensitivity is produced by the residual contribution (side lobes) of the baseband component. As shown in Fig. 2, it is not completely removed from the system by the balanced photodetection. In this case, sensitivity achieves values around 40 and $45 \mathrm{~dB}$. In the second range from 5.5 to $8 \mathrm{~mm}$, the noise contribution is just limited by the noise floor of the VNA. Therefore, a maximum value of $60 \mathrm{~dB}$ is obtained for this second range.

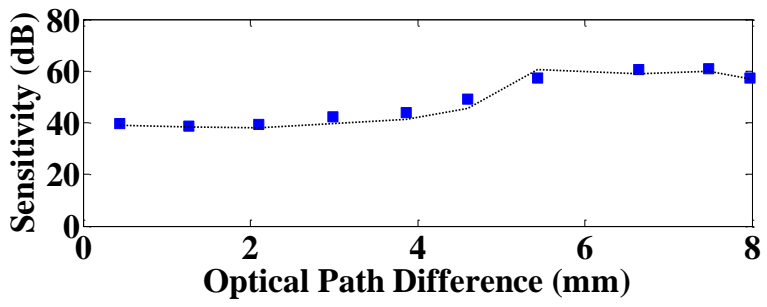

Fig. 5. Experimental results of the system sensitivity (dots) when different OPDs are set in the VDL. Theoretical simulation added in dashed line.

\section{CONCLUSION}

We have proposed a MWP-LCI scheme with the introduction of the SCM technique to provide higher scanning rates for LCI applications compared to previous MWP-LCI approaches operating in the RF domain. By multiplexing different subcarriers in the modulation stage, the sweep time to obtain the electrical transfer function of the structure can be considerably reduced. When demultiplexing is performed, a reconstruction of the electrical transfer function can be carried out by the combination of the individual acquisitions which are simultaneously measured.

Experimentally, a two-layered sample scenario is considered to demonstrate the system viability. The proposed approach not only takes profit from the inherent stability of $\mathrm{RF}$ domain systems but also permits to reduce the scanning rate for LCI applications. Since our MWP-LCI system operates under small signal regime, the crosstalk between RF subcarriers is minimized. In this way, a linear dependence between the central frequency of each resonance with the OPDs set in the interferometer. An excellent agreement is found between experimental results and theoretical predictions. A total OPD range of $8 \mathrm{~mm}$ is achieved in this case, with a quasi-invariant resolution of $120 \mu \mathrm{m}$. Finally, the sensitivity of the system is analyzed. For this, the noise produced by the structure is also measured. A maximum sensitivity value of $60 \mathrm{~dB}$ is demonstrated which is limited by the noise floor of the VNA.

\section{REFERENCES}

[1] D Huang, E. A. Swanson, C. P. Lin, J. S. Schuman, W. G. Stinson, W. Chang, M. R. Hee, T. Flotte, K. Gregory, C. A. Puliafito, "Optical Coherence Tomography," Science, vol. 254, pp. 1178-1181, Nov. 1991.

[2] A. Gh. Podoleanu, "Optical Coherence Tomography," J. of Microscopy, vol. 247, no. 3, pp. 209-219, 2012.

[3] H. Liang, B. Peric, M. Hughes, A. Podoleanu, M. Spring and D. Saunders, "Optical coherence tomography for art conservation and archaeology" in Proceedings SPIE 6618, 2007.

[4] P. Nandi, Z. Chen, A. Witkowska, W. J. Wadsworth, T. A. Birks, Jo. C. Knight, "Characterization of a photonic crystal fiber mode converter using low coherence interferometry," Opt. Lett. vol. 34, no. 7, pp. 11231125,2009

[5] P.V. Volkov, A.V. Goryunov, A.Yu. Luk'yanov, A.D. Tertyshnik, N.A. Baidakova, I.A. Luk'yanov, "Fiber-optic temperature sensor based on low-coherence interferometry without scanning," Optik, vol. 124, no. 15, pp. 1982-1985, 2013.

[6] J. Capmany and D. Novak, "Microwave Photonics combines two worlds," Nat. Photonics, vol. 1, pp. 319-330, 2007.

[7] W. Li and J. Yao, "Microwave and Terahertz Generation Based on Photonically Assisted Microwave Frequency Twelvetupling With Large Tunability," IEEE Photon. J., vol. 2, no. 6, pp. 954-959, 2010.

[8] A. L. Ricchiuti, J. Hervás, D. Barrera, S. Sales and J. Capmany, "Microwave Photonics Filtering Technique for Interrogating a VeryWeak Fiber Bragg Grating Cascade Sensor," IEEE Photon. J, vol. 6, no. 6, pp. 1-10, 2014.

[9] C. R. Fernandez-Pousa, J. Mora, H. Maestre, P. Corral, "Radiofrequency low-coherence interferometry," Opt. Lett., vol. 39, no. 12, pp. 3634-3637, Jun. 2014.

[10] J. Benitez; M. Bolea; J. Mora, "High performance low coherence interferometry using SSB modulation," IEEE Photon. Technol. Lett., vol. 29, no. 1, pp. 90-93, 2017.

[11] M. Bolea, J. Mora and J. Capmany, "A novel MWP proposal for lowcoherence interferometry applications," in $M W P$, Paphos, 2015, pp. 1-4.

[12] K. Goda, and B. Jalali, "Dispersive Fourier transformation for fast continuous single-shot measurements," Nat. Photonic, vol. 7, pp. 102112, 2013.

[13] J. Benitez, M. Bolea and J. Mora, "Real-time Microwave Photonic technique for Low-Coherence Interferometry applications," 2016 18th International Conference on Transparent Optical Networks (ICTON), Trento, 2016, pp. 1-4.

[14] J. Mora, B. Ortega, A. Díez, J. L. Cruz, M. V. Andrés, J. Capmany, and D. Pastor, "Photonic Microwave Tunable Single-Bandpass Filter Based on a Mach-Zehnder Interferometer," J. Lightw. Technol., vol. 24, no. 7, pp. 2500-2509, 2006.

[15] J. Capmany, A. Ortigosa-Blanch, J. Mora, A. Ruiz-Alba, W. Amaya and A. MartÍnez, "Analysis of Subcarrier Multiplexed Quantum Key Distribution Systems: Signal, Intermodulation, and Quantum Bit Error Rate," IEEE Journal of Selected Topics in Quantum Electronics, vol. 15, no. 6, pp. 1607-1621, 2009. 